\title{
PERFIL DE IDOSOS VOTANTES NO RIO GRANDE DO SUL
}

\section{Profile of older adult voters in the state of Rio Grande do Sul}

\author{
Ângelo José Gonçalves Bósa , Walessa Signorinil, Cletiane Dias Rodriguesc, Caroline Trennepohld
}

\begin{abstract}
OBJETIVOS: Pouco se sabe sobre a participação cívica da pessoa idosa no Brasil, por isso propôs-se investigar os fatores associados à participação dos idosos nas eleições presidenciais de 2010. MÉTODOS: Foram analisados os dados do inquérito epidemiológico, transversal e observacional realizado no período de 2010 a 2011, com 7.315 idosos identificados por visita domiciliar usando amostragem aleatória dos setores censitários de 59 cidades do Estado do Rio Grande do Sul, Brasil. Análise de regressão logística usou como variável dependente a informação dicotômica se o idoso teria ou não votado na eleição de 2010 (votou) e como variáveis independentes: sociodemográficas, hábitos de vida, dificuldades de locomoção, autopercepção de saúde e número de morbidades. RESULTADOS: Independente da idade, 71\% dos entrevistados idosos votou. O percentual de idosos entre 60 e 69 anos que votaram foi maior do que o observado na população geral. Homens e mulheres apresentaram frequências semelhantes. Foram fatores significativos para ter votado nas eleições de 2010: idade, escolaridade, autopercepção de saúde, morbidades, característica da locomoção, segurança, ler jornais, assistir noticiário na televisão, atividades comunitárias, atividade física regular, atividade econômica e conhecer o Estatuto do Idoso. CONCLUSÃO: Idosos votantes no Rio Grande do Sul são mais jovens, casados, com ótima ou boa autopercepção de saúde, com maior número de morbidades, sem dificuldades de locomoção, se mantém informados, conhecem o Estatuto do Idoso e são ativos, tanto social, quando física e economicamente. PALAVRAS-CHAVE: participação social; envelhecimento; política; saúde pública.
\end{abstract}

OBJECTIVES: Little is known about the civic participation of the elderly in Brazil, so it was proposed to investigate the factors associated with the participation of the elderly in the presidential election of 2010. METHODS: We analyzed the data from an epidemiological cross-sectional and observational survey conducted in 2010-2011, involving 7,315 elderly identified by home visits to randomly selected census sectors of 59 cities in the State of Rio Grande do Sul, Brazil. Logistic regression analysis used the dichotomous dependent variable if the elderly not voted in the 2010 election (voted), and the independent variables were: sociodemographic, lifestyle, mobility difficulties, self-rated health and number of comorbidities. RESULTS: Regardless of age, 71\% of older adult respondents voted. The percentage of elderly between 60 and 69 years who voted was higher than that observed in the general population. Men and women had similar frequencies. Significant factors to vote in the 2010 elections were: age, education, self-rated health, morbidity, mobility, safety, reading newspapers, watching news on television, community activities, regular physical activity, economic activity and knowledge of the Statute of the Elderly. CONCLUSION: Elderly voters in Rio Grande do Sul are younger, married, with excellent or good self-rated health, with more morbidities, without walking difficulties, remain informed, know the Statute of the Elderly and are social, physical and economically active.

KEYWORDS: social participation; aging; politics; public health.

aGeriatra, Professor Adjunto do Instituto de Geriatria e Gerontologia da Pontifícia Universidade Católica do Rio Grande do Sul (PUCRS) Porto Alegre (RS), Brasil.

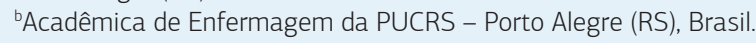

'Bacharel em Administração de Empresas, PUCRS - Porto Alegre (RS), Brasil.

dEnfermeira, PUCRS - Porto Alegre (RS), Brasil.

Dados para correspondência

Ângelo José Gonçalves Bós - Avenida Ipiranga, 6681 - CEP: 90669-900 - Porto Alegre (RS), Brasil - E-mail: angelo.bos@pucrs.br 


\section{INTRODUÇÃO}

O envelhecimento da população brasileira tem despertado o interesse da sociedade em busca de uma população idosa socialmente ativa. A Organização Mundial da Saúde (OMS) tem preconizado o envelhecimento ativo que indica que, mais do que manter-se ativo fisicamente, as pessoas idosas devem buscar manter-se socialmente ativos. ${ }^{1} \mathrm{~A}$ participação cívica das pessoas ajuda a promover uma sociedade mais inclusiva e eleger representantes que apoiam as necessidades da população. A OMS, preocupada não somente com o envelhecimento mas também com a urbanização da população idosa nas sociedades contemporâneas, principalmente em países em desenvolvimento como o Brasil, tem discutido também a necessidade de desenvolverem-se "cidades amigas do idoso". Uma cidade amiga do idoso é aquela que adapta as suas estruturas, tornando-se mais acessível e urbanamente acolhedora de seus idosos, promovendo sua inclusão e usufruindo do potencial que esse contingente populacional representa. ${ }^{1}$ Nesse contexto há a necessidade de eleger governantes sensíveis a essa política.

Pouco se sabe sobre a participação cívica da pessoa idosa no Brasil. Estudos internacionais demonstram que as pessoas idosas são as que mais votam proporcionalmente. ${ }^{2}$ Entretanto, a legislação brasileira difere de muitos países, visto que o voto é obrigatório até os 70 anos e não obrigatório após essa idade. Dados do Tribunal Superior Eleitoral (TSE) sobre as eleições presidenciais de 2010 demonstram que quase todos os idosos entre 60 e 79 anos estavam aptos a votar. O comparecimento da população geral durante as eleições de 2010 variou entre 76 e $86 \%$ nas diferentes unidades da Federação. ${ }^{3}$ Não se observou na literatura científica brasileira nenhuma publicação sobre os fatores que levam as pessoas idosas brasileiras a votar. Em recente pesquisa realizada em 2011, 7.315 idosos residentes em diferentes cidades do Estado do Rio Grande do Sul foram questionados sobre diversas características a respeito das suas atividades e condições de vida relacionadas às observadas no relatório da OMS "Guia global: cidade amiga do idoso". O estudo observou que a participação em atividades sociais e cívicas (se votou ou não) foi um fator protetor para quedas nos idosos. ${ }^{4}$ Propomos então analisar os dados dessa pesquisa com o objetivo de investigar os fatores associados à participação ou não dos idosos nas eleições presidenciais de 2010.

\section{MÉTODOS}

O presente estudo é um estudo transversal observacional de análise secundária de dados. Analisa os dados do inquérito epidemiológico realizado no período de 2010 a 2011, com 7.315 idosos de 59 cidades do Estado do Rio Grande do Sul, Brasil, denominado Perfil dos Idosos do RS (PIRS), realizado pelo Instituto de Geriatria e Gerontologia da Pontifícia Universidade Católica do Rio Grande do Sul (IGG-PUCRS) em parceria com a Escola de Saúde Pública do Rio Grande do Sul (ESP/RS). O estudo foi baseado no "Guia global: cidade amiga do idoso", publicado pela OMS, ${ }^{5}$ e objetivou identificar e avaliar o perfil dos idosos e o ambiente onde vivem, através de uma pesquisa representativa dessa faixa etária no Estado. Dados metodológicos foram publicados recentemente. ${ }^{4}$ Os idosos foram identificados por visita domiciliar a setores censitários aleatoriamente selecionados em 59 cidades do Estado do Rio Grande do Sul.

Como variável dependente foi utilizada a informação dicotômica se o idoso teria ou não votado (votou), em 2010. Foram escolhidas variáveis independentes: sociodemográficas (sexo, estado conjugal e faixa etária), hábitos de vida (ler jornal, assistir noticiário na televisão ou rádio, acessar Internet diariamente, sair de casa e conversar com amigos semanalmente, realizar atividade física regular, participar mensalmente de atividades sociais e comunitárias), dificuldades de locomoção, autopercepção de saúde e número de morbidades (doenças crônicas autorreferidas). As características das variáveis independentes foram expressas em frequências para a variável votou e a sua associação testada pelo teste do $\chi^{2}$, exceto para o número de morbidades, que foi expresso em média e desvio padrão, e as possíveis diferenças comparadas pela análise de variância (ANOVA). Índice de significância menor do que $5 \%$ foi considerado estatisticamente significativo e entre 5 e 10\% como indicativo de significância. As variáveis independentes foram incluídas em um modelo inicial de regressão logística. A retirada sistemática dos fatores menos significativos proporcionou alcançar o modelo final de regressão logística onde todos os fatores significativos ou com indicativo de significância para a predição de votação foi obtido. Somente o modelo final foi mostrado, que incluiu a variável sexo mesmo sem ser significativa. $O$ projeto PIRS foi aprovado pelos Comitês de Ética em Pesquisa da ESP/RS (0481/2009) e da PUCRS (09/04931).

\section{RESULTADOS}

Foram analisados todos os 7.315 registros do banco de dados do PIRS, 71\% das pessoas idosas haviam votado na eleição de 2010. Na Tabela 1 é possível observar as características sociodemográficas e clínicas dos participantes e o fato de terem ou não votado. A proporção de pessoas idosas do sexo masculino foi $2 \%$ menor do que o feminino, 
Tabela 1 Distribuição dos idosos conforme as características sociodemográficas e clínicas e o fato de ter votado (Perfil dos Idosos do Rio Grande do Sul, 2011)

\begin{tabular}{|c|c|c|c|c|}
\hline & & & & \\
\hline & $\operatorname{sim}_{n(\%)}$ & $\begin{array}{l}\text { Não } \\
\text { n (\%) }\end{array}$ & n (\%) & Valor p \\
\hline Gênero & & & & 0,1122 \\
\hline Feminino & $2647(69,9)$ & $1139(30,1)$ & $3786(51,8)$ & \\
\hline Masculino & $2527(71,6)$ & $1002(28,4)$ & $3529(48,2)$ & \\
\hline Faixa Etária & & & & $<0,0001$ \\
\hline $60-69 a$ & $3480(91,3)$ & $331(8,7)$ & $3811(52,1)$ & \\
\hline $70-79 a$ & $1392(54,9)$ & $1145(45,1)$ & $2537(34,7)$ & \\
\hline $80-89 a$ & $291(32,3)$ & $610(67,7)$ & $901(12,3)$ & \\
\hline $90 \mathrm{a}+$ & $11(16,7)$ & $55(83,3)$ & $66(0,9)$ & \\
\hline Escolaridade & & & & $<0,0001$ \\
\hline Analfabeto & $338(50,2)$ & $335(49,8)$ & $673(9,2)$ & \\
\hline Alfabetizado & $621(67,5)$ & $299(32,5)$ & $920(12,6)$ & \\
\hline Primário incompleto & $650(64,0)$ & $366(36,0)$ & $1016(13,9)$ & \\
\hline Primário completo & $2016(71,5)$ & $802(28,5)$ & $2818(38,5)$ & \\
\hline Ginasial completo & $948(82,3)$ & $204(17,7)$ & $1152(15,7)$ & \\
\hline Secundário ou superior & $601(81,7)$ & $135(18,3)$ & $736(10,1)$ & \\
\hline Estado conjugal & & & & $<0,0001$ \\
\hline Casado & $2779(78,7)$ & $753(21,3)$ & $3532(48,3)$ & \\
\hline Separado & $553(76,6)$ & $169(23,4)$ & $722(9,9)$ & \\
\hline Solteiro & $265(66,2)$ & $135(33,8)$ & $400(5,5)$ & \\
\hline Viúvo & $1379(57,8)$ & $1005(42,2)$ & $2384(32,6)$ & \\
\hline Autopercepção saúde & & & & $<0,0001$ \\
\hline Ótima/boa & $3894(76,9)$ & $1171(23,1)$ & $5065(69,2)$ & \\
\hline Regular & $1058(58,3)$ & $756(41,7)$ & $1814(24,8)$ & \\
\hline Péssima/má & $183(51,4)$ & $173(48,6)$ & $356(4,9)$ & \\
\hline Memória comprometida & $149(43,7)$ & $192(56,3)$ & $341(4,7)$ & $<0,0001$ \\
\hline $\begin{array}{l}\text { Número de morbidades } \\
\text { (média } \pm \text { desvio padrão) }\end{array}$ & $1,47 \pm 1,570$ & $1,37 \pm 1,500$ & $1,41 \pm 1,522$ & 0,0017 \\
\hline Dificuldade de locomoção* & $305(45,5)$ & $365(54,5)$ & $670(9,2)$ & $<0,0001$ \\
\hline Falta de companhia* & $162(65,8)$ & $84(34,2)$ & $246(3,4)$ & 0,0871 \\
\hline Falta de segurança* & $1083(73,4)$ & $392(26,6)$ & $1475(20,2)$ & 0,0109 \\
\hline Total & $5174(70,7)$ & $2141(29,3)$ & 7315 (100) & \\
\hline
\end{tabular}

*Dificuldade para sair de casa; a: anos.

mas essa proporção foi maior entre os votantes, não havendo associação significativa. Quanto maior a faixa etária, maior foi a proporção de não votantes $(p<0,0001)$. Apenas $9 \%$ dos entrevistados eram analfabetos. Participantes com níveis mais elevados de escolaridade apresentaram maior proporção de votantes $(\mathrm{p}<0,001)$. Casados e separados apresentaram as maiores proporções de votantes $(\mathrm{p}<0,001)$. A maioria dos participantes referiu apresentar ótima ou boa autopercepção de saúde, essa variável foi significativamente associada com votação. $\mathrm{O}$ número médio de morbidades referidas pelos entrevistados foi significativamente maior entre as pessoas idosas votantes. Pessoas idosas que apresentavam memória 
comprometida, em teste de memorizar três palavras, ou referiram ter dificuldade de locomoção apresentaram menor proporção de votantes, sendo essa associação significativa. A proporção de participantes que referiram ter dificuldade de sair de casa por falta de companhia foi de $66 \%$, sendo menor do que a proporção total de votantes (71\%), essa associação com votação apresentou indicativo de significância $(p=0,087)$. As pessoas idosas que referiram ter dificuldade de sair de casa por falta de segurança apresentaram maior proporção de votantes, sendo essa associação significativa.

A Tabela 2 apresenta a distribuição dos participantes quanto aos hábitos de vida, a participação sociocomunitária e a ocupação conforme o fato de ter ou não votado na eleição de 2010. Apresentaram maior proporção e associação significativa com votação as seguintes atividades: acessar a Internet pelo menos uma vez por semana, assistir noticiário na TV, ler jornal, conhecer o Estatuto do Idoso, realizar atividade comunitária, atividade física regular, conversar com amigos semanalmente, trabalhar como autônomo, empregado formal ou informal e cuidar de outras pessoas. Realizar atividades voluntárias obteve indicativo de significância com maior proporção de votantes. Participar de grupo de idosos foi indicativo de significância com votação sendo a proporção menor do que o esperado, o mesmo acontecendo entre os participantes que referiram não realizar nenhuma atividade, sendo essa associação significativa.

As variáveis que obtiveram associação ou diferença significativa ou indicativo de significância, além do

Tabela 2 Distribuição dos participantes quanto aos hábitos de vida, participação sociocomunitária e ocupação conforme o fato de ter votado na eleição de 2010 presidencial (Perfil dos Idosos do Rio Grande do Sul, 2011)

\begin{tabular}{|c|c|c|c|c|}
\hline & \multicolumn{2}{|c|}{$\begin{array}{l}\text { Votou } \\
\text { n (\%) } \\
\end{array}$} & \multirow{2}{*}{$\begin{array}{l}\text { Total } \\
\text { n (\%) }\end{array}$} & \multirow{2}{*}{ Valor $p$} \\
\hline & $\begin{array}{c}\text { Sim } \\
n(\%)\end{array}$ & $\begin{array}{l}\text { Não } \\
\text { n (\%) }\end{array}$ & & \\
\hline Acessa Internet & $746(87,1)$ & $111(12,9)$ & $857(11,7)$ & $<0,0001$ \\
\hline Assiste noticiário na TV & $4908(71,7)$ & $1939(28,3)$ & $6847(93,6)$ & $<0,0001$ \\
\hline Lê jornal & $2601(76,9)$ & $780(23,1)$ & $3381(46,2)$ & $<0,0001$ \\
\hline Ouve rádio & $3742(71,1)$ & $1524(28,9)$ & $5266(72,0)$ & 0,3225 \\
\hline Conhece o Estatuto do Idoso & $1610(78,2)$ & $449(21,8)$ & $2059(28,1)$ & $<0,0001$ \\
\hline Atividade comunitária & $1045(80,3)$ & $257(19,7)$ & $1302(17,8)$ & $<0,0001$ \\
\hline Atividade física regular & $1424(79,2)$ & $373(20,8)$ & $1797(24,6)$ & $<0,0001$ \\
\hline Conversa com amigos & $4543(72,3)$ & $1739(27,7)$ & $6282(85,9)$ & $<0,0001$ \\
\hline \multicolumn{5}{|l|}{ Ocupação } \\
\hline Atividades voluntárias & $66(79,5)$ & $17(20,5)$ & $83(1,1)$ & 0,0768 \\
\hline Trabalho remunerado & $430(90,5)$ & $45(9,5)$ & $475(6,5)$ & $<0,0001$ \\
\hline Trabalho informal & $303(80,8)$ & $72(19,2)$ & $375(5,1)$ & $<0,0001$ \\
\hline Atividades domésticas & $1383(72,1)$ & $536(27,9)$ & $1919(26,2)$ & 0,1338 \\
\hline Cuida de outras pessoas & $27(93,1)$ & $2(6,9)$ & $29(0,4)$ & 0,0079 \\
\hline Atividades de lazer & $572(70,5)$ & $239(29,5)$ & $811(11,1)$ & 0,8937 \\
\hline Atividades cívicas & $59(68,6)$ & $27(31,4)$ & $86(1,2)$ & 0,6628 \\
\hline Grupo de idosos & $52(61,9)$ & $32(38,1)$ & $84(1,1)$ & 0,0737 \\
\hline Outro grupo & $2(40,0)$ & $3(60,0)$ & $5(0,1)$ & 0,1308 \\
\hline Empregador & $10(83,3)$ & $2(16,7)$ & $12(0,2)$ & 0,3369 \\
\hline Autônomo & $131(90,3)$ & $14(9,7)$ & $145(2,0)$ & $<0,0001$ \\
\hline Outra atividade & $40(76,9)$ & $12(23,1)$ & $52(0,7)$ & 0,3247 \\
\hline Nenhuma atividade & $2502(66,6)$ & $1253(33,4)$ & $3755(51,3)$ & $<0,0001$ \\
\hline Total & $5174(70,7)$ & $2141(29,3)$ & 7315 (100) & \\
\hline
\end{tabular}


gênero, foram incluídas no modelo inicial de regressão logística múltipla para estudar a associação entre as variáveis e a chance de ter votado. A retirada sistemática das variáveis menos significativas foi realizada até que gênero e somente as variáveis significativas ou com indicativo se significância permanecessem no modelo final. Na Tabela 3 observamos o resultado do modelo final de regressão logística múltipla para a associação das variáveis de estudo e a chance do idoso ter votado. Observamos que a variável gênero não foi significativa e que os participantes do sexo masculino tiveram apenas 5\% maior chance de ter votado na eleição de 2010 comparados com os participantes do sexo feminino. Pessoas com 60 a 69 anos tinham significativamente mais chances de votarem do que as pessoas entre 70 e 79 anos, contrariamente quanto maior a faixa etária, menor a chance de votar, sendo todas as associações significativas. Os nonagenários foram os que apresentaram menor chance de votar, sendo $80 \%$ menores chances de votar do que as pessoas idosas com 70 a 79 anos. Quanto à escolaridade, as pessoas idosas alfabetizadas foram usadas como referência no estudo da associação entre escolaridade e a chance de terem votado. Observamos que quanto maior o grau de escolaridade, maior foram as chances de votar, principalmente após o ginasial completo, sendo significativo para os com secundário completo ou superior, que obtiveram 35\% mais chance de votar $(\mathrm{p}=0,032)$. Quanto pior a autopercepção de saúde das pessoas idosas, menor foi a chance de votar, sendo todas as comparações significativas. As pessoas idosas que apresentavam dificuldade de locomoção como motivo para não sair de casa apresentavam $37 \%$ menores chances de terem votado, sendo essa associação significativa $(\mathrm{p}<0,001)$. Pessoas idosas que não saíam de casa por falta de segurança tiveram mais chance de votar do que as outras, sendo essa associação muito significativa $(p=0,006)$. Ler jornais e assistir noticiário na televisão foram fatores significativamente positivos para a participação da pessoa idosa na eleição para Presidente da República de 2010.

\section{DISCUSSÃO}

Nos dados relatados neste estudo, observou-se que quanto maior a faixa etária, menor foi o número de idosos votantes, o que corrobora os dados do TSE (2014) que indica que o total de 1,7 milhão do eleitorado do RS é de pessoas idosas, e desses, somente $15 \%$ tem mais de 79 anos, estando a maioria dos idosos (56\%) na faixa etária de 60 a 69 anos, portanto poderiam se fazer presentes nas urnas eleitorais. ${ }^{6}$
Tabela 3 Resultado da modelo final da regressão logística para as chances de ter votado (Perfil dos Idosos do Rio Grande do Sul, 2011, 2011)

\begin{tabular}{|c|c|c|}
\hline & $\begin{array}{l}\text { Razão de chance } \\
\text { (IC95\%) }\end{array}$ & Valor $p$ \\
\hline \multicolumn{3}{|l|}{ Gênero } \\
\hline Feminino (Ref.) & 1 & \\
\hline Masculino & $1,05(0,93-1,20)$ & 0,4217 \\
\hline \multicolumn{3}{|l|}{ Faixa etária } \\
\hline 60 a 69 anos & $7,71(6,64-8,97)$ & $<0,0001$ \\
\hline 70 a 79 anos (Ref.) & 1 & \\
\hline 80 a 89 anos & $0,42(0,35-0,50)$ & $<0,0001$ \\
\hline 90 anos ou mais & $0,22(0,11-0,45)$ & $<0,0001$ \\
\hline \multicolumn{3}{|l|}{ Escolaridade } \\
\hline Analfabeto & $0,63(0,49-0,81)$ & $<0,0001$ \\
\hline Alfabetizado (Ref.) & 1 & \\
\hline Primário incompleto & $0,95(0,76-1,20)$ & 0,6801 \\
\hline Primário completo & $0,95(0,78-1,16)$ & 0,6005 \\
\hline Ginasial completo & $1,09(0,84-1,4)$ & 0,5219 \\
\hline Secundário ou superior & $1,20(0,90-1,60)$ & 0,2147 \\
\hline \multicolumn{3}{|l|}{ Estado Conjugal } \\
\hline Casado (Ref.) & 1 & \\
\hline Separado/divorciado & $0,80(0,64-1,00)$ & 0,0549 \\
\hline Solteiro & $0,57(0,43-0,75)$ & $<0,0001$ \\
\hline Viúvo & $0,74(0,64-0,85)$ & $<0,0001$ \\
\hline Morbidades & $1,11(1,07-1,17)$ & $<0,0001$ \\
\hline \multicolumn{3}{|l|}{ Autopercepção de saúde } \\
\hline Ótima ou boa (Ref.) & 1 & \\
\hline Regular & $0,56(0,49-0,65)$ & $<0,0001$ \\
\hline Péssima ou má & $0,41(0,31-0,54)$ & $<0,0001$ \\
\hline Memória comprometida & $0,63(0,47-0,84)$ & 0,0015 \\
\hline Ocupação, autônomo & $1,92(1,04-3,57)$ & 0,0385 \\
\hline Ocupação, nenhuma & $0,83(0,73-0,95)$ & 0,0062 \\
\hline Falta de companhia* & $1,45(1,04-2,02)$ & 0,0284 \\
\hline Dificuldade de locomoção & $0,62(0,50-0,76)$ & $<0,0001$ \\
\hline Assiste noticiário na TV & $1,29(1,00-1,66)$ & 0,0524 \\
\hline Lê jornal & $1,19(1,04-1,36)$ & 0,0111 \\
\hline Atividade comunitária & $1,28(1,06-1,53)$ & 0,0090 \\
\hline Atividade física regular & $1,37(1,17-1,60)$ & $<0,0001$ \\
\hline Conversa com amigos & $1,25(1,05-1,48)$ & 0,0135 \\
\hline
\end{tabular}

IC95\%: intervalo de confiança de 95\%; Ref.: referência; *dificuldade para não sair de casa. 
A relação entre nível de escolaridade e proporção de votantes perdeu significância quando ajustado por faixa etária. Isso provavelmente se deve ao fato de pessoas mais idosas apresentarem nível educacional menor. ${ }^{7}$ A história política do país mostra que o vínculo entre educação e voto não representou a oferta de educação de qualidade à população brasileira. Representou, ao contrário, a exclusão política das maiorias sociais, que no Brasil continuaram analfabetas por muito tempo. ${ }^{8}$

Estado conjugal foi um importante fator associado à maior chance de ter votado. Casados tiveram maior chance de votar que os solteiros, separados e viúvos, mesmo na análise ajustada. Talvez reflita a maior responsabilidade que os casados ainda assumam frente à família. Afirmação essa que necessita ser confirmada em estudos futuros.

Idosos com maior número de morbidades tiveram mais chances de votar. Talvez porque o fato de terem maior número de doenças faz com que frequentem com maior assiduidade os serviços de saúde, observando as dificuldades existentes, motivando-os a agir de uma forma que tente resolver essa questão. $\mathrm{O}$ voto é um dos poucos instrumentos que a população dispõe na tentativa de resolver os seus problemas públicos.

Idosos com pior percepção de saúde votam menos, talvez por não se sentirem em condições clínicas de comparecerem às urnas. Esse fenômeno parece contraditório ao fato anterior, que relacionou o maior número de morbidades à maior chance de ter votado. Alvarenga et al., 9 em seu estudo, mostram que idosos com autopercepção ruim da sua saúde tem 12 vezes mais chances de apresentar sintomas depressivos, muito comuns em idosos, do que os que a julgavam muito boa ou boa. O humor deprimido, o interesse diminuído por outras atividades, sentimento de inutilidade e culpa, entre outros, são sintomas da depressão em idosos, o que pode justificar a não participação na votação eleitoral. ${ }^{10}$ Ser economicamente ativo foi um fator positivamente associado à chance de votar, mesmo ajustando por idade, escolaridade e gênero.

Dificuldade de locomoção, como motivo que dificulta o idoso sair de casa, foi um fator negativamente associado à chance de votar. Essa restrição talvez demonstre a dificuldade no acesso dos idosos ao local de votação ou no entorno do domicílio. Essa dificuldade de acesso pode ser tanto pela falta de manutenção de calçadas ou vias ou por questões de segurança. ${ }^{4}$

Falta de companhia para sair de casa foi inesperadamente fator positivo para ir às urnas eletrônicas. Esperava-se ser um fator negativo à votação, nos fazendo refletir na ausência do suporte familiar e social do idoso. ${ }^{11}$ Entretanto, parece refletir como um fator motivacional a busca por uma modificação da sociedade pelo voto. Contrariamente, a falta de segurança como fator que dificulta o idoso a sair de casa demonstrou-se não significativo na análise múltipla pela regressão logística, evidenciando que outros fatores foram mais importantes do que esse.

Assistir noticiário na televisão diminuiu sua significância na análise múltipla, mantendo-se como indicativo de significância. Por outro lado, ler jornal foi fator significativo, mesmo ajustando por outras variáveis como escolaridade e faixa etária. O resultado demonstra que a leitura é uma maneira mais ativa de se manter atualizado, sendo a televisão uma forma passiva de informação.

Idosos mais ativos social e fisicamente demonstram-se mais predispostos a serem também civicamente ativos, demonstrando que a interação social é um importante fator motivacional para o exercício do seus direitos. Esse último fator é corroborado pelo fato dos idosos conhecedores do Estatuto do Idoso apresentarem maior chance de terem votado, mesmo ajustando pelo nível educacional e faixa etária. Giacomin et al. ${ }^{12}$ referem que para o cidadão que vive em um país que envelhece rápido e intensamente, sua atuação deve ser pautada no exercício de sua cidadania, para além da obrigação de votar.

Ressalta-se a escassez de estudos sobre a presente temática, além de se identificar a ausência de maiores dados publicados pelo poder público, ignorando, assim, o tema abordado. Sugere-se, portanto, a maior produção científica para se melhor conhecer a importância da participação da pessoa idosa na votação eleitoral, visto ser cada vez mais importante a sua participação na sociedade.

\section{CONCLUSÃO}

Este estudo é o primeiro a analisar quantitativamente o perfil epidemiológico dos idosos que votam. Não foi possível encontrar na literatura suporte teórico aos achados. Os resultados demonstraram associação significativa entre votar e faixa etária, escolaridade, estado civil, autopercepção de saúde, morbidades, dificuldade de locomoção, segurança, assistir noticiário na TV, ler jornal, conhecer o Estatuto do Idoso, realizar atividade comunitária, atividade física regular e ser economicamente ativo.

\section{CONFLITO DE INTERESSES}

Os autores informam não haver conflito de interesses. 


\section{REFERÊNCIAS}

1. Organização Mundial da Saúde. Guia global: cidade amiga do idoso. Genebra: Organização Mundial da Saúde; 2008.

2. Dubrow JK. Do political parties represent women, the poor, and the elderly? Int J Sociol. 2012; 42(1):78-86.

3. Brasil. Tribunal Superior Eleitoral. Repositório de dados eleitorais. /www.tse.jus.br/hotSites/pesquisas-eleitorais/eleitorado.html (Acessado em: 24/06/2013)

4. Pereira GN, Morsch P, Lopes DGC, Trevisan MD, Ribeiro A, Navarro JHN, et al. Fatores socioambientais associados à ocorrência de quedas em idosos. Ciênc Saúde Coletiva. 2013; 18(12):3507-14.

5. Marques RO. Política pública para o idoso: um estudo da cidadania no grupo Cabelo de Neve do Programa Conviver em Campinas Grande-PB [dissertação]. Natal: Universidade Federal do Rio Grande do Norte; 2010.

6. Brasil. Tribunal Superior Eleitoral. Estatísticas de eleitorado. /www.tse.jus.br/eleitor/estatisticas-de-eleitorado (Acessado em: 17/08/2014)
7. Diniz BSO, Volpe FM, Tavares AR. Nível educacional e idade no desempenho no Miniexame do Estado Mental em idosos residentes na comunidade. Rev Psiquiatr Clín. 2007; 34(1):13-17.

8. Dore R, Ribeiro S. Cidadania política e voto do analfabeto no Brasil. Políticas Públicas. 2009; 2(2):84-99.

9. Alvarenga MRM, Oliveira MAC, Faccenda O, Cerchiari EAN, Amendola F. Sintomas depressivos em idosos assistidos pela Estratégia Saúde da Família. Cogitare Enferm. 2010; 15(2):217-24.

10. Rodrigues NL. Depressão, ansiedade, outros transtornos afetivos e suicídio. In: Freitas EV, Py L, editor. Tratado de Geriatria e Gerontologia. Rio de Janeiro: Guanabara Koogan; 2011.

11. Inouye K, Barham EJ, Pedrazzani ES, Pavarini SCI. Percepções de suporte familiar e qualidade de vida entre idosos segundo a vulnerabilidade social. Psicol Reflex Crít. 2010; 23(3):582-92.

12. Giacomin KC, Couto EC. O caráter simbólico dos direitos referentes à velhice na constituição federal e no estatuto do idoso. Rev Kairós. 2013; 16(2):141-60. 